\title{
Metric properties of some special $p$-adic series expansions
}

\author{
by
}

\section{Arnold Knopfmacher and John Knopfmacher (Johannesburg)}

1. Introduction. Let $\mathbb{Q}$ be the field of rational numbers, $p$ a prime number and $\mathbb{Q}_{p}$ the completion of $\mathbb{Q}$ with respect to the $p$-adic valuation ||$_{p}$ defined on $\mathbb{Q}$ by

$$
|0|_{p}=0 \quad \text { and } \quad|A|_{p}=p^{-a} \quad \text { if } A=p^{a} r / s, \quad \text { where } p \nmid r, p \nmid s .
$$

Then $\mathbb{Q}_{p}$ is the field of $p$-adic numbers with $p$-adic valuation ||$_{p}$, the extension of the original valuation on $\mathbb{Q}$ (cf. Koblitz [12] or Schikhof [19]).

It is well known that every $A \in \mathbb{Q}_{p}$ has a unique series representation $A=\sum_{n=v(A)}^{\infty} c_{n} p^{n}, c_{n} \in\{0,1,2, \ldots, p-1\}$. In the discussion below we call the finite series $\langle A\rangle=\sum_{v(A) \leq n \leq 0} c_{n} p^{n}$ the fractional part of $A$. Then $\langle A\rangle \in S_{p}$, where we define $S_{p}=\left\{\langle A\rangle: A \in \mathbb{Q}_{p}\right\} \subset \mathbb{Q}$.

This set $S_{p}$ is not multiplicatively or additively closed. The function $\langle A\rangle$ and set $S_{p}$ have been used in the study of certain types of $p$-adic continued fractions by Mahler [14], Ruban [17] and Laohakosol [13] in particular.

Recently the fractional part $\langle A\rangle$ was used by the present authors [7], [8] to derive some new unique series expansions for any element $A \in \mathbb{Q}_{p}$, including in particular analogues of certain "Sylvester", "Engel" and "Lüroth" expansions of arbitrary real numbers into series with rational terms (cf. [16], Chap. IV). It turns out that $p$-adic and real Lüroth series may be regarded in some sense as algorithmic relatives of continued fractions, and there is some interest in studying possible parallels between the algorithms or digits inducing them. In the direction of metric and asymptotic results concerning digits, various analogies of this kind were previously established, especially by Jager and de Vroedt [5] and Salát [18] for real Lüroth series, and by Ruban [17] for $p$-adic continued fractions, in comparison with classical theorems of Khinchin (see e.g. [2], [6]) for real continued fractions. (In these developments, Haar measure for $p$-adic numbers replaces Lebesgue measure for real numbers.)

The main aim of the present paper is to state or derive some similar metric and asymptotic results for the $p$-adic Lüroth-type expansions re- 
ferred to above. Here, as in other areas such as e.g. transcendence and diophantine approximation theory (cf. Sprindžuk [20]), there are also parallels with results in the partly analogous but different context of Laurent series over finite fields; see Paysant-Le Roux and Dubois [15] and the present authors [9], [10]. Consequently, many of the steps below are given only in outline, together with references to fuller parallel arguments where appropriate. (We thank an anonymous referee for some helpful comments.)

2. Lüroth-type algorithm and ergodic properties. The Lüroth-type expansion (see (2.1) below) of a $p$-adic number $A \in \mathbb{Q}_{p}$ was derived in [7] from the following algorithm for the "digits" $a_{n}=a_{n}(A) \in S_{p}$ :

Define $a_{0}=\langle A\rangle$ and $A_{1}=A-a_{0}$ and observe that

$$
a_{0}=c \in S_{p} \Leftrightarrow v(A-c) \geq 1 \Leftrightarrow A-c \in X_{p},
$$

where $X_{p}=p \mathbb{Z}_{p}$ is the maximal ideal in the ring $\mathbb{Z}_{p}$ of all $p$-adic integers, i.e. $p$-adic numbers of order $\geq 0$. If $A_{n} \neq 0(n \geq 1)$ has already been defined, inductively define

$$
a_{n}=\left\langle 1 / A_{n}\right\rangle \quad \text { and } \quad A_{n+1}=\left(a_{n}-1\right)\left(a_{n} A_{n}-1\right),
$$

so that $v\left(a_{n}\right) \leq-1$ for $n \geq 1$. If any $A_{m}=0$, or $a_{m}=0$, stop the algorithm.

This algorithm leads (cf. [7]) to a finite or convergent (relative to ||$_{p}$ ) expansion

$$
A=a_{0}+\frac{1}{a_{1}}+\sum_{n \geq 2} \frac{1}{a_{1}\left(a_{1}-1\right) \ldots a_{n-1}\left(a_{n-1}-1\right) a_{n}},
$$

which is unique for $A$ subject to the stated condition on the digits $a_{n}$. Another way of looking at it is in terms of operators $a$ and $T$ (where $a$ : $\left.X_{p} \backslash\{0\} \rightarrow S_{p}, T: X_{p} \rightarrow X_{p}\right)$ such that $a(x)=\langle 1 / x\rangle, T(0)=0$ and otherwise $T(x)=(a(x)-1)(x a(x)-1)$. Then for $x=A_{1} \in X_{p}$ we have $a_{1}=a_{1}(x)=a(x)$ and more generally $a_{n}=a_{n}(x)=a_{1}\left(T^{n-1} x\right)$ if $0 \neq$ $T^{n-1} x \in X_{p}$.

Although the conclusions of the next theorem are sharpened in Section 3 below it seems at least worth sketching briefly how they can also be deduced from the Ergodic Theorem, after proving that $x \in X_{p} \Rightarrow T(x) \in X_{p}$ and that the resulting operator $T: X_{p} \rightarrow X_{p}$ is ergodic relative to Haar measure $\mu$ on $X_{p}$.

Theorem 1. (i) For any given $k \in S_{p}$ with $v(k) \leq-1$, and all $x \in X_{p}$ outside a set of Haar measure 0 , the digit value $k$ has asymptotic frequency

$$
\lim _{n \rightarrow \infty} \frac{1}{n} \#\left\{r \leq n: a_{r}(x)=k\right\}=|k|_{p}^{-2} .
$$


(ii) For all $x \in X_{p}$ outside a set of Haar measure 0 there exists a single "Khinchin-type" constant

$$
\lim _{n \rightarrow \infty}\left|a_{1}(x) \ldots a_{n}(x)\right|_{p}^{1 / n}=p^{p /(p-1)} .
$$

(iii) For all $x \in X_{p}$ outside a set of Haar measure 0,

$$
\left|x-w_{n}\right|_{p}=p^{(-2 p /(p-1)+o(1)) n} \quad \text { as } n \rightarrow \infty,
$$

where

$$
w_{n}=w_{n}(x)=\sum_{r=1}^{n} \frac{\lambda_{r-1}}{a_{r}}, \quad \lambda_{0}=1, \lambda_{r}=\frac{1}{a_{1}\left(a_{1}-1\right) \ldots a_{r}\left(a_{r}-1\right)} .
$$

For this and later theorems, a convenient description of the Haar measure $\mu$ on $X_{p}=p \mathbb{Z}_{p}$ is given in Sprindžuk [20], pp. 67-70. In particular, $\mu(C)=$ $p^{-r}$ for any "circle", "disc" or "ball"

$$
C=C\left(x, p^{-r-1}\right):=\left\{y \in \mathbb{Q}_{p}:|y-x|_{p} \leq p^{-r-1}\right\}
$$

of radius $p^{-r-1}$. So $\mu\left(X_{p}\right)=1$, since $X_{p}=C\left(0, p^{-1}\right)$.

Now note that every "digit" $a(x)$ lies in the set $S_{p}^{*}:=\{\langle A\rangle: v(A) \leq-1\}$. For any given digits $k_{1}, \ldots, k_{n} \in S_{p}^{*}$, let

$$
I_{n}=I_{n}\left(k_{1}, \ldots, k_{n}\right):=\left\{x \in X_{p}: a_{1}(x)=k_{1}, \ldots, a_{n}(x)=k_{n}\right\}
$$

and call $I_{n}$ a basic (Lüroth) cylinder of rank $n$. Also let $I_{0}=X_{p}$.

The Lüroth-type expansion (2.1) of any $x \in I_{n}$ then has the form

$$
x=w_{n}+\lambda_{n} \sum_{r>n} \frac{1}{a_{n+1}\left(a_{n+1}-1\right) \ldots a_{r-1}\left(a_{r-1}-1\right) a_{r}},
$$

where

$$
\lambda_{0}=1, \quad \lambda_{r}=\frac{1}{k_{1}\left(k_{1}-1\right) \ldots k_{r}\left(k_{r}-1\right)} \quad \text { for } 1 \leq r \leq n
$$

and

$$
w_{n}=\sum_{r=1}^{n} \frac{\lambda_{r-1}}{k_{r}} .
$$

Thus $x=w_{n}+\lambda_{n} T^{n}(x)=\psi_{n}\left(T^{n}(x)\right)$, if $\psi_{n}=\psi_{n}\left(k_{1}, \ldots, k_{n}\right): X_{p} \rightarrow I_{n}$ is defined by $\psi_{n}(y)=w_{n}+\lambda_{n} y\left(y \in X_{p}\right)$. The "linear-type" map $\psi_{n}$ is then 1-1 onto, with inverse map $T^{n}: I_{n} \rightarrow X_{p}$. In particular, $I_{n}=\operatorname{Im}\left(\psi_{n}\right)=$ $w_{n}+\lambda_{n} X_{p}$. Since $X_{p}=C\left(0, p^{-1}\right)$, it then follows that $I_{n}=C\left(w_{n}, p^{-1}\left|\lambda_{n}\right|_{p}\right)$ and has Haar measure $\mu\left(I_{n}\right)=p^{-v\left(\lambda_{n}\right)}=\left|\lambda_{n}\right|_{p}$. Hence

$$
\mu\left(I_{n}\right)=\frac{1}{\left|k_{1}\left(k_{1}-1\right) \ldots k_{n}\left(k_{n}-1\right)\right|_{p}}=\frac{1}{\left|k_{1} \ldots k_{n}\right|_{p}^{2}},
$$

since $v(k)=v(k-1)$ for $v(k) \leq-1$. 
More generally, for any $r_{1}<\ldots<r_{n}$, we obtain

$$
\mu\left\{x \in X_{p}: a_{r_{1}}(x)=k_{1}, \ldots, a_{r_{n}}(x)=k_{n}\right\}=\left|k_{1} \ldots k_{n}\right|_{p}^{-2} .
$$

In particular, $\mu\left\{x \in X_{p}: a_{r}(x)=k\right\}=|k|_{p}^{-2}$ for any $r \geq 1$ and $k \in S_{p}^{*}$. Thus the digit functions are identically distributed and independent random variables relative to $\mu$.

Now, in a standard way quite similar to that followed by Jager and de Vroedt [5] for real Lüroth series, one can deduce that $T$ is measurepreserving and ergodic. (In fact, the stronger Bernoulli property for $T$ could be approached along lines analogous to some given in [1].)

Theorem 1 and some further conclusions then follow by making special choices for integrable functions $f$ in the ergodic formula

$$
\lim _{n \rightarrow \infty} \frac{1}{n} \sum_{r=1}^{n} f\left(T^{r-1} x\right)=\int_{X_{p}} f d \mu \quad \text { a.e. }
$$

For example, part (i) of Theorem 1 follows from consideration of the characteristic function $f_{k}$ of a basic cylinder $I_{1}(k)$. Alternatively, use of the function $\widehat{f}(\cdot)=\log _{p}\left|a_{1}(\cdot)\right|_{p}$ leads to the limit

$$
\lim _{n \rightarrow \infty} \frac{1}{n} \sum_{r=1}^{n} \log _{p}\left|a_{r}(x)\right|_{p}=\int_{X_{p} \backslash\{0\}} \widehat{f} d \mu=\frac{p}{p-1} \quad \text { a.e., }
$$

and this implies part (ii) of Theorem 1 . The same function $\widehat{f}$ may be used in the deduction of part (iii), in combination with the following inequalities analogous to some appearing for Laurent series in [10]:

$$
1-\sum_{r=1}^{n+1} \log _{p}\left|a_{r}(x)\right|_{p}^{2} \leq \log _{p}\left|x-w_{n}\right|_{p} \leq-1-\sum_{r=1}^{n} \log _{p}\left|a_{r}(x)\right|_{p}^{2} .
$$

The function $\widehat{f}$ may also be used to show that the operator $T$ has entropy

$$
h(T)=-\lim _{n \rightarrow \infty} \frac{1}{n} \log _{e} \mu\left(I_{n}\right)=\frac{2 p \log _{e} p}{p-1} .
$$

Lastly, it is interesting to note that, in contrast to (2.5), a truncation argument involving the function $\tilde{f}(\cdot)=\left|a_{1}(\cdot)\right|_{p}$ leads to the conclusion

$$
\lim _{n \rightarrow \infty} \frac{1}{n} \sum_{r=1}^{n}\left|a_{r}(x)\right|_{p}=\infty \quad \text { a.e. }
$$

3. Sharper asymptotic estimates. The fact that the $p$-adic Lürothtype digit functions $a_{r}(\cdot)$ define identically distributed and independent random variables on $X_{p}$ paves the way for the introduction of methods and 
results of probability theory, which lead to sharper and deeper results than those considered earlier.

In the first place, the law of the iterated logarithm and the central limit theorem (cf. Theorems 3.16/17 in Galambos [4]) yield:

Theorem 2. Let $A_{n, k}(x)=\#\left\{r \leq n: a_{r}(x)=k\right\}$. Then for almost all $x \in X_{p}$,

Further, for any real $z$,

$$
\limsup _{n \rightarrow \infty} \frac{A_{n, k}(x)-n|k|_{p}^{-2}}{\sqrt{n \log \log n}}=\sqrt{2|k|_{p}^{-2}\left(1-|k|_{p}^{-2}\right)} .
$$

$$
\begin{aligned}
\lim _{n \rightarrow \infty} \mu\left\{x \in X_{p}: A_{n, k}(x)-n|k|_{p}^{-2}<\frac{z}{|k|_{p}} \sqrt{n\left(1-|k|_{p}^{-2}\right)}\right\} & \\
& =\frac{1}{\sqrt{2 \pi}} \int_{-\infty}^{z} e^{-u^{2} / 2} d u .
\end{aligned}
$$

Now define a sequence $\left(t_{n}\right)$ of independent random variables $t_{n}$ on $X_{p}$ by

$$
t_{n}(x)= \begin{cases}v\left(a_{n}(x)\right) & \text { if }\left|a_{n}(x)\right|_{p} \leq n^{2} \\ 0 & \text { otherwise }\end{cases}
$$

Then the expected value

$$
E\left(t_{n}\right)=\sum_{p^{r} \leq n^{2}} p^{-2 r} r(p-1) p^{r}=E\left(v\left(a_{n}(\cdot)\right)\right)+O\left(\frac{\log n}{n^{2}}\right),
$$

and a similar calculation of $E\left(t_{n}^{2}\right)$ and the variance $\operatorname{var}\left(t_{n}\right)$ leads to the estimate

$$
B_{n}:=\sum_{r=1}^{n} \operatorname{var}\left(t_{r}\right)=\frac{p n}{(p-1)^{2}}+O(1) \quad \text { as } n \rightarrow \infty .
$$

Next, since $t_{n}(x) \leq 2 \log _{p} n=o\left(\sqrt{B_{n} / \log \log B_{n}}\right)$, the law of the iterated logarithm implies

$$
\limsup _{n \rightarrow \infty} \frac{\sum_{r=1}^{n} t_{r}-\sum_{r=1}^{n} E\left(t_{r}\right)}{\sqrt{2 B \log \log B_{n}}}=1 \quad \text { a.e. }
$$

Hence

$$
\limsup _{n \rightarrow \infty} \frac{\sum_{r=1}^{n} t_{r}-\sum_{r=1}^{n} E\left(v\left(a_{r}(\cdot)\right)\right)}{\sqrt{2 \frac{p}{(p-1)^{2}} n \log \log n}}=1 \quad \text { a.e. }
$$

Now let $U_{n}=\left\{x \in X_{p}: t_{n}(x) \neq v\left(a_{n}(x)\right)\right\}$. Then

$$
\mu\left(U_{n}\right)=\sum_{|k|_{p}>n^{2}}|k|_{p}^{-2}<\frac{1}{n^{2}},
$$


and the Borel-Cantelli lemma yields $\mu\left(\lim \sup _{n \rightarrow \infty} U_{n}\right)=0$. Thus, for almost all $x \in X_{p}$, there exists $n_{0}(x)$ with $t_{n}(x)=v\left(a_{n}(x)\right)$ for $n \geq n_{0}(x)$. Therefore (3.3) now implies:

Theorem 3. For almost all $x \in X_{p}$,

$$
\limsup _{n \rightarrow \infty} \frac{\sum_{r=1}^{n} v\left(a_{r}(x)\right)-c_{1} n}{\sqrt{n \log \log n}}=\sqrt{2 c_{2}},
$$

where $c_{1}=p /(p-1), c_{2}=p /(p-1)^{2}$. Hence as $n \rightarrow \infty$,

$$
\left|a_{1}(x) \ldots a_{n}(x)\right|_{p}^{1 / n}=p^{p /(p-1)}+O\left(\sqrt{\frac{\log \log n}{n}}\right) \quad \text { a.e. }
$$

The next theorem sharpens the last part of Theorem 1 above:

Theorem 4. If $w_{n}=w_{n}(x)$ is defined as in Theorem 1(iii) then

$$
\limsup _{n \rightarrow \infty} \frac{v\left(x-w_{n}\right)+2 p n /(p-1)}{\sqrt{n \log \log n}}=\frac{\sqrt{8 p}}{p-1} \quad \text { a.e. }
$$

Hence

$$
\frac{1}{n} v\left(x-w_{n}\right)=-\frac{2 p}{p-1}+O\left(\sqrt{\frac{\log \log n}{n}}\right) \quad \text { a.e. }
$$

By symmetry as in Feller [3], p. 205, Theorem 3 leads to

$$
\liminf _{n \rightarrow \infty} \frac{\sum_{r=1}^{n} v\left(a_{r}^{2}(x)\right)-2 p n /(p-1)}{\sqrt{n \log \log n}}=-2 \sqrt{2 c_{2}} \quad \text { a.e. }
$$

In combination with (2.6) above, this implies Theorem 4.

4. Average and individual estimates for digits. By (2.8) above, the average

$$
\frac{1}{n} \sum_{r=1}^{n}\left|a_{r}(x)\right|_{p} \rightarrow \infty \quad \text { a.e. on } X_{p}
$$

as $n \rightarrow \infty$. Theorem 5 estimates this average in probability over $X_{p}$ :

Theorem 5. For any fixed $\varepsilon>0$,

$$
\lim _{n \rightarrow \infty} \mu\left\{x \in X_{p}:\left.\frac{1}{n \log _{p} n}\left|\sum_{r=1}^{n}\right| a_{r}(x)\right|_{p}-(p-1) \mid>\varepsilon\right\}=0,
$$

i.e.

$$
\frac{1}{n \log _{p} n} \sum_{r=1}^{n}\left|a_{r}(x)\right|_{p} \rightarrow p-1 \quad \text { in probability over } X_{p} \text {. }
$$


Pr o of. Consider the truncation method of Feller [3], Chapter 10, $\S 2$, as applied to the random variables $U_{r}, V_{r}(r \leq n)$ defined by

$$
\begin{array}{llll}
U_{r}(x)=\left|a_{r}(x)\right|_{p}, & V_{r}(x)=0 & & \text { if }\left|a_{r}(x)\right|_{p} \leq n \log _{p} n, \\
U_{r}(x)=0, & V_{r}(x)=\left|a_{r}(x)\right|_{p} & & \text { if }\left|a_{r}(x)\right|_{p}>n \log _{p} n .
\end{array}
$$

In that case

$$
\begin{aligned}
\mu\left\{x \in X_{p}\right. & \left.:\left.\frac{1}{n \log _{p} n}\left|\sum_{r=1}^{n}\right| a_{r}(x)\right|_{p}-(p-1) \mid>\varepsilon\right\} \\
\leq & \mu\left\{x:\left|U_{1}+\ldots+U_{n}-(p-1) n \log _{p} n\right|>\varepsilon n \log _{p} n\right\} \\
& +\mu\left\{x: V_{1}+\ldots+V_{n} \neq 0\right\},
\end{aligned}
$$

and

$$
\begin{aligned}
\mu\left\{x: V_{1}+\ldots+V_{n} \neq 0\right\} & \leq n \mu\left\{x:\left|a_{1}(x)\right|_{p}>n \log _{p} n\right\} \\
& =n \sum_{|k|_{p}>n \log _{p} n}|k|_{p}^{-2}<1 / \log _{p} n=o(1) .
\end{aligned}
$$

Next $E\left(U_{1}+\ldots+U_{n}\right)=n E\left(U_{1}\right)$ and $\operatorname{var}\left(U_{1}+\ldots+U_{n}\right)=n \operatorname{var}\left(U_{1}\right)$, where

$$
\begin{aligned}
E\left(U_{1}\right) & =\sum_{|k|_{p} \leq n \log _{p} n}|k|_{p}^{-1}=(p-1) \log _{p}\left(\left[n \log _{p} n\right]\right) \\
& \sim(p-1) \log _{p} n \quad \text { as } n \rightarrow \infty
\end{aligned}
$$

and

$$
\operatorname{var}\left(U_{1}\right)<E\left(U_{1}^{2}\right)=\sum_{|k|_{p} \leq \log _{p} n} 1<p n\left(\log _{p} n\right) .
$$

Theorem 5 then follows from an application of (4.3) and (4.4) to Chebyshev's inequality:

$$
\mu\left\{x:\left|U_{1}+\ldots+U_{n}-n E\left(U_{1}\right)\right|>\varepsilon n E\left(U_{1}\right)\right\} \leq \frac{n \operatorname{var}\left(U_{1}\right)}{\left(\varepsilon n E\left(U_{1}\right)\right)^{2}}=o(1) .
$$

Note that the conclusion of Theorem 5 is not valid with probability one, since Theorem 3.13 in Galambos [4] implies that either

$$
\limsup _{n \rightarrow \infty} \frac{1}{n \log _{p} n} \sum_{r=1}^{n}\left|a_{r}(x)\right|_{p}=\infty \quad \text { a.e. }
$$

or

$$
\liminf _{n \rightarrow \infty} \frac{1}{n \log _{p} n} \sum\left|a_{r}(x)\right|_{p}=0 \quad \text { a.e. }
$$

Regarding estimates for individual digits, now consider: 
THEOREM 6. Given any positive increasing function $\psi(n)$ of $n$,

$$
\left|a_{n}(x)\right|_{p}=O(\psi(n)) \text { a.e. } \Leftrightarrow \sum_{n=1}^{\infty} 1 / \psi(n)<\infty .
$$

In fact, $\left|a_{n}(x)\right|_{p}=O(\psi(n))$ is false a.e. if the series diverges.

Proof. Let $V_{n}=\left\{x \in X_{p}:\left|a_{n}(x)\right|_{p}>\psi(n)\right\}$. Since $\mu\left\{x: a_{n}(x)=k\right\}$ $=|k|_{p}^{-2}$ by $(2.3)$, it follows that

$$
\mu\left(V_{n}\right)=\sum_{|k|_{p}>\psi(n)}|k|_{p}^{-2} \leq p / \psi(n) .
$$

If $\sum_{n=1}^{\infty} \psi(n)^{-1}<\infty$, then the Borel-Cantelli lemma (cf. [4], p. 36) yields $\mu\left(\lim \sup V_{n}\right)=0$. Hence $\left|a_{n}(x)\right|_{p}>\psi(n)$ for at most finitely many $n$, for almost all $x \in X_{p}$. Thus $\left|a_{n}(x)\right|_{p}=O(\psi(n))$ a.e.

If $\sum_{n=1}^{\infty} \psi(n)^{-1}$ diverges, the Abel-Dini theorem (Knopp [11], p. 290) implies that there exists a positive increasing function $\theta(n)$ with $\theta(n) \rightarrow \infty$ as $n \rightarrow \infty$ such that $\sum_{n=1}^{\infty} \psi(n)^{-1} \theta(n)^{-1}$ also diverges. Then let $W_{n}=$ $\left\{x \in X_{p}:\left|a_{n}(x)\right|_{p}>\psi(n) \theta(n)\right\}$. The independence of the random variables $a_{n}$ implies the independence of the sets $W_{n}$. Also

$$
\sum_{n=1}^{\infty} \mu\left(W_{n}\right)=\sum_{n=1}^{\infty} \sum_{|k|_{p}>\psi(n) \theta(n)}|k|_{p}^{-2}>\frac{1}{p} \sum_{n=1}^{\infty} \frac{1}{\psi(n) \theta(n)}=\infty .
$$

Thus the Borel-Cantelli lemma yields $\mu\left(\lim \sup W_{n}\right)=1$, and so $\left|a_{n}(x)\right|_{p}>$ $\psi(n) \theta(n)$ holds with probability one, for infinitely many $n$. Thus $\left|a_{n}(x)\right|_{p}=$ $O(\psi(n))$ is false a.e.

Corollary. For almost all $x \in X_{p}$,

$$
\limsup _{n \rightarrow \infty} \frac{\log \left|a_{n}(x)\right|_{p}-\log n}{\log \log n}=1 .
$$

Proof. Theorem 6 implies that $\left|a_{n}(x)\right|_{p}=O\left(n(\log n)^{\alpha}\right)$ a.e. for any $\alpha>1$, while $\left|a_{n}(x)\right|_{p}=O\left(n(\log n)^{\beta}\right)$ is false a.e. for any $\beta \leq 1$. The corollary then follows by choosing $\alpha=1+\varepsilon, \beta=1-\varepsilon(\varepsilon>0)$.

(Note that the corresponding lower limit is not finite a.e., since (2.3) earlier shows that $\left|a_{n}(x)\right|_{p}$ can take any particular constant value $p^{N}(N \geq$ 1) for all $n$, and all $x$ in a set of positive measure.)

\section{References}

[1] J. Barrionuevo, R. M. Burton, K. Dajani and C. Kraaikamp, Ergodic properties of generalized Lüroth series, Acta Arith. 74 (1996), 311-327.

[2] P. Billingsley, Ergodic Theory and Information, Wiley, 1965. 
[3] W. Feller, An Introduction to Probability Theory and its Applications, Vol. 1, 3rd ed., Wiley, 1968.

[4] J. Galambos, Representations of Real Numbers by Infinite Series, Springer, 1976.

[5] H. Jager and C. de Vroedt, Lüroth series and their ergodic properties, Nederl. Akad. Wetensch. Proc. Ser. A 72 (1969), 31-42.

[6] A. Y. Khintchine, Metrische Kettenbruchprobleme, Compositio Math. 1 (1935), 361-382.

[7] A. Knopfmacher and J. Knopfmacher, Series expansions in p-adic and other non-archimedean fields, J. Number Theory 32 (1989), 297-306.

[8] —, - Infinite series expansions for p-adic numbers, ibid. 41 (1992), 131-145.

[9] - - - Metric properties of algorithms inducing Lüroth series expansions of Laurent series, Astérisque 209 (1992), 237-246.

[10] J. Knopfmacher, Ergodic properties of some inverse polynomial series expansions of Laurent series, Acta Math. Hungar. 60 (1992), 241-246.

[11] K. Knopp, Theory and Application of Infinite Series, Dover, 1990.

[12] N. Koblitz, p-adic Numbers, p-adic Analysis, and Zeta-Functions, 2nd ed., Springer, 1984.

[13] Y. Laohakosol, A characterization of p-adic Ruban continued fractions, J. Austral. Math. Soc. A 39 (1985), 300-305.

[14] K. Mahler, Zur Approximation p-adischer Irrationalzahlen, Nieuw Arch. Wisk. 18 (1934), 22-34.

[15] R. Paysant-Le Roux and E. Dubois, Étude métrique de l'algorithme de JacobiPerron dans un corps de séries formelles, C. R. Acad. Sci. Paris A 275 (1972), 683-686.

[16] O. Perron, Irrationalzahlen, Chelsea, 1951.

[17] A. A. Ruban, Some metric properties of p-adic numbers, Siberian Math. J. 11 (1970), 176-180.

[18] T. Salát, Zur metrischen Theorie der Lürothschen Entwicklungen der reellen Zahlen, Czechoslovak Math. J. 18 (1968), 489-522.

[19] W. H. Schikhof, Ultrametric Calculus, Cambridge University Press, 1984.

[20] V. G. Sprindžuk, Mahler's Problem in Metric Number Theory, Amer. Math. Soc., 1969.

A. Knopfmacher

Department of Computational \&

Applied Mathematics

University of the Witwatersrand

Johannesburg, 2050, South Africa

E-mail: arnoldk@gauss.cam.wits.ac.za
J. Knopfmacher Department of Mathematics University of the Witwatersrand Johannesburg, 2050, South Africa 